\title{
Traffic Flow Models for Road Network with Intersections
}

\author{
Zhongwen Chen \\ Department of Science and Technology, Huanggang Normal University, Huanggang 438000, \\ Hubei Province, China \\ zhongwenchen@foxmail.com
}

Keywords: Traffic flow model; Road network with intersections; Multi-agent

\begin{abstract}
This paper discussed the influence of the self-driving car on the traffic flow, and presented the traffic-density-velocity traffic flow (TDVTF) model and cellular automata model based on multi-agent. From the law of conservation, the single lane, multi-lane TDVTF models and TDVTF models for road network with intersections are established. As for multi-lane TDVTF model, the function of self-driving car proportion with respect to self-driving car density and human- driving car density are obtained.
\end{abstract}

\section{Introduction}

Traffic capacity is limited in many regions of the United States due to the number of lanes of roads. For example, in the Greater Seattle area drivers experience long delays during peak traffic hours because the volume of traffic exceeds the designed capacity of the road networks. This is particularly pronounced on Interstates 5, 90, and 405, as well as State Route 520, the roads of particular interest for this problem.

Self-driving, cooperating cars have been proposed as a solution to increase capacity of highways

without increasing number of lanes or roads. The behavior of these cars interacting with the existing

traffic flow and each other is not well understood at this point.

With the development of socio-economic and the acceleration of motorization, Highway traffic jams have become more and more serious. To alleviate the existing highway traffic jam problems without increasing number of lanes or roads, the self-driving cars have been proposed [1-3] and experimented [4-6]. The self-driving cars are believed to create many more benefits, such as to relieve drivers from many tedious tasks, to increase road safety, to improve the traffic flow, and to decrease environment suffers. Along with that, self-driving cars have been widely concerned in recent years.

Various papers dedicated to the microscopic traffic flow models of the intelligent vehicles have appeared in the open literature, which gives the impacts on dynamic traffic flow features [7]. To our knowledge, there do not exist researches on the exact effects on the traffic flows of allowing selfdriving, cooperating cars, which motivates this study. We would like to depict the effect changes as the percentage of self-driving cars increases by constructing simple mathematical models in combination with the data analysis.

\section{The Model}

To investigate the effect of the self-driving car on the traffic flow, a differentiable model of acceleration and safe-distance is developed firstly to based on different information communication systems. Then a cellular model based on multi-agents is implemented according to the differential model of the acceleration and safe-distance. Based on the background and the problems we faced, self-driving cars are joined in the existing vehicles. We study the changes of traffic flow by changing the proportion of self-driving cars, deduce the percentage when the traffic flow achieves balance, solve tipping point problem where performance changes markedly, and analyze the situation when to set separate lane for self-driving cars. The models are built to analyze the effects 
on traffic flow of the number of lanes, peak or average traffic volume and percentage of vehicles using self-driving, cooperating cars.

Case 1: Single car. The differences between the operation of self-driving cars and humandriving cars are listed below. As for a single car, the difference is mainly reflected in the following four aspects:

To improve the traffic safety, the safe distance of the self-driving car is larger than the critical safe distance, and the number of lane changing is reduced as much as possible; During driving process, human-drivers always have a certain reaction time before each decision, while it does not exist for a self-driving car; Due to the high sensitivity of the sensor, a self-driving car is more sensitive to the surrounding environment than a human-driver, which helps it make more appropriate decisions in time; The driving decision of a human-driver tends to depend on his own judgment, which is usually greatly affected by personal psychological factors, while a self-driving car tends to make more objective judgments.

Case 2: Multiple cars. As for multiple cars, the difference mainly exists in the cooperation mechanism. The multi-self-driving cars always communicate to each other via Vehicle-to-Vehicle (V2V) or Vehicle-to-Infrastructure (V2I) communications to enhance the perception of the driving environment and to assist cooperative vehicles under a common goal. However, due to the limited view, human-driver can only observe the running conditions of the vehicles around. Thus they are unable to access to real-time information of the traffic network timely and make appropriate and timely decisions, resulting in a traffic jam or even accidents. Under the cooperating system control aiming at providing the best driving condition, the movements of self-driving cars tend to an equilibrium state and the traffic flow tends to a balance.

If each vehicle is regarded as an agent, the vehicles in the whole traffic network will form a multi-agent network topology. Due to the lack of information exchange, the human-driving car can only be connected with the adjacent nodes, but the self-driving car could even be connected with each node in the network.

TDVTF Model. The four roads we considered throughout the paper constitute a network. To analyze the traffic flow, we first build a traffic flow model on a single lane based on the law of conservation of traffic, which yields the expression of traffic flow at peaking time and other times [3] respectively. Subsequently, by utilizing the exchange rate of different lanes [3], the model is extended to multi-lane model. Furthermore, the multi-lane model is extended to network model in consideration of the crossroads impact.

Single Lane TDVTF Model. We take a lane element dx (between 1 and 2 in Fig. 1) along stream direction on the unidirectional continuous lane. There is no exit or entrance in the road element, which means the traffic flow is a constant. Denote the number of vehicles run perpendicular to the road per unit time by the traffic flow q. By the basic traffic flow formula, we have

$\mathrm{q}=\rho \mathrm{v}$

where $v$ denotes the average speed and $\rho$ denotes the average car density.

Similarly, take a time element $d t$. During this time interval, the traffic density changes from $\rho$ to $\rho+d \rho$. And the corresponding traffic flow changes from $q$ to $q+d q$. Applying the Law of Conservation of Mass, we obtain

$$
\frac{\partial \rho}{\partial t}+\frac{\partial q}{\partial x}=0
$$

If the traffic density is moderate, then we adopt the speed-density linear model introduced by Green shilds that

$$
v=v_{\max }\left(1-\frac{\rho}{\rho_{\max }}\right)
$$

where $v_{\max }$ is free stream velocity and $\rho_{\max }$ is obstructed density. Substituting the above equation into (1), it is readily to obtain the traffic flow model in normal times 


$$
q_{1}=\rho_{1} v_{\max }\left(1-\frac{\rho_{1}}{\rho_{\max }}\right)
$$

If the traffic density is relatively large, then we adopt the speed-density logarithmic model introduced by Greenberg that

$$
v=v_{\max } \ln \left(\frac{\rho_{\max }}{\rho}\right)
$$

In the same line, we obtain the traffic flow model in peaking times

$$
q_{2}=\rho_{2} v_{\max } \ln \left(\frac{\rho_{\max }}{\rho}\right)
$$

Now we consider $n$ lanes in the same direction on a road. Then each lane satisfies the conservation equation, i.e.

$$
\begin{gathered}
\frac{\partial \rho_{1}}{\partial t}+\frac{\partial q_{1}}{\partial x}=b_{1} \\
\frac{\partial \rho_{2}}{\partial t}+\frac{\partial q_{2}}{\partial x}=b_{2} \\
\cdots \\
\frac{\partial \rho_{n}}{\partial t}+\frac{\partial q_{n}}{\partial x}=b_{n}
\end{gathered}
$$

where $q_{\mathrm{i}}(x, t), \rho_{\mathrm{i}}(x, t)$ and $b_{\mathrm{i}}(x, t)(i=1,2, \ldots, n)$ are the traffic flow, the traffic density and in the pure exchange rate of lane i respectively. $b_{\mathrm{i}}(x, t)>0$ indicates net entry and $b_{\mathrm{i}}(x, t)<0$ indicates net departure. Hence, we have

$$
b_{i}=\alpha_{i, j-1}\left[\left(\rho_{i-1}-\rho_{i}\right)-\left(\rho_{i-1,0}-\rho_{i, 0}\right)\right]+\alpha_{i, j+1}\left[\left(\rho_{i+1}-\rho_{i}\right)-\left(\rho_{i+1,0}-\rho_{i, 0}\right)\right],
$$

where $\alpha$ is the sensitivity coefficient whose unit is $s^{-1}$ and $\rho_{\mathrm{i} 0}$ is equilibrium density of lane $i$. Additionally, since the traffic system is closed and traffic flow is conservative, we have

$$
\sum_{i=1}^{n} b_{i}=0
$$

(4) Road Network TDVTF Model

In the above models, we didn't consider inflow and outflow process. At a crossroad, the traffic flow changes. Regarding that the inflow and outflow, influence of time lag, and sensitivity coefficient all changes with density, we improve the model to be

$$
\begin{aligned}
& \frac{\partial \rho_{1}}{\partial t}+\frac{\partial q_{1}}{\partial x}=b_{1} \\
& \frac{\partial \rho_{2}}{\partial t}+\frac{\partial q_{2}}{\partial x}=b_{2}
\end{aligned}
$$




$$
\frac{\partial \rho_{n}}{\partial t}+\frac{\partial q_{n}}{\partial x}=b_{n}+g
$$

where $g(x, t)$ is the net flow rate of the far right lane. Similarly, $g(x, t)>0$ indicates entry and $g(x$, $t)<0$ indicates departure. Hence, we have

$$
\begin{aligned}
& b_{i}=\alpha_{i, j-1}\left\{\left[\left(\rho_{i-1}(x, t-\tau)-\rho_{i}(x, t-\tau)\right]-\left(\rho_{i-1,0}-\rho_{i, 0}\right)\right\}\right. \\
& +\alpha_{i, j+1}\left[\left(\rho_{i+1}(x, t-\tau)-\rho_{i}(x, t-\tau)\right)-\left(\rho_{i+1,0}-\rho_{i, 0}\right)\right]
\end{aligned}
$$

where $\tau$ is the interaction lag time.

\section{Conclusions}

In this paper, the problem of analyzing the influence of the self-driving car on the traffic flow, and presented the traffic-density-velocity traffic flow (TDVTF) model and cellular automata model based on multi-agent are studied, and the single lane, multi-lane TDVTF models and TDVTF models for road network with intersections are established based on the law of conservation. In the multi-lane TDVTF model, the function of self-driving car proportion with respect to self-driving car density and human- driving car density are obtained. The results shown that self-driving car proportion only depends on one of the two densities.

\section{Acknowledgments}

This work was supported by the 2013 Humanities and Social Science Planning Fund Project of Ministry of Education (No. 13YJA630010), and the Outstanding Young Innovative Group Project of Hubei Province (No.T201415).

\section{References}

[1] W.J. Li, Simulation of Urban Vehicle Lane Changing Model Based on Cellular Automata, Jilin University, 2015.

[2] J.C. Dai, X. Li, Microscopic traffic flow simulation model based on multi-Agent, Chinese Science Bulletin 21 (2009) 3380-3389.

[3] J. Treiterer, Investigation of Traffic Dynamic by Aerial Photogrammetry Techniques, Final Report EES278, 1975.

[4] G.H. Bham, R.F. Benekohal, A high fidely traffic simulation model based on cellular automata and car-following concepts, Transport Res Part C-Emer 12 (2004) 1-32.

[5] W. Wang, X.C. Guo, Traffic Engineering, Southeast University Press, Nanjing, 2000.

[6] C. Urmson, W.R. Whittaker, Self-driving cars and the urban challenge, IEEE Intelligent Systems 23 (2008) 66-68.

[7] M. Wang, Cooperative car-following control: Distributed algorithm and impact on moving jam features, IEEE Transactions on Intelligent Transportation Systems 17 (2016) 1459-1471. 